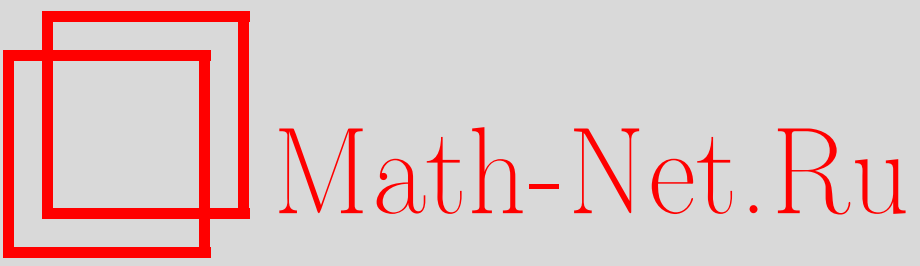

В. В. Власов, Н. А. Раутиан, Представление решений вольтерровых интегродифференциальных уравнений с дробно-экспоненциальными ядрами, Итоги науки и техн. Сер. Соврем. мат. и ее прил. Темат. обз., 2021, том 194, 92-106

DOI: https://doi.org/10.36535/0233-6723-2021-194-92-106

Использование Общероссийского математического портала Math-Net.Ru подразумевает, что вы прочитали и согласны с пользовательским соглашением

http://www.mathnet.ru/rus/agreement

Параметры загрузки:

IP : 18.234 .156 .22

26 апреля 2023 г., 18:07:27 


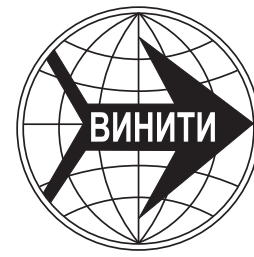

ИТОГИ НАУКИ И ТЕХНИКИ.

Современная математика и ее приложения.

Тематические обзоры.

Том 194 (2021). С. 92-106

DOI: 10.36535/0233-6723-2021-194-92-106

УДК 517.968 .72

\title{
ПРЕДСТАВЛЕНИЕ РЕШЕНИЙ ВОЛЬТЕРРОВЫХ ИНТЕГРО-ДИФФЕРЕНЦИАЛЬНЫХ УРАВНЕНИЙ С ДРОБНО-ЭКСПОНЕНЦИАЛЬНЫМИ ЯДРАМИ
}

\author{
(c) 2021 г. $\quad$ В. В. ВЛАСОВ, Н. А. РАУТИАН
}

\begin{abstract}
АннотАция. Целью настоящей работы является изучение асимптотического поведения решений интегро-дифференциальных уравнений на основе спектрального анализа их символов. Для этого мы получаем представления сильных решений указанных уравнений в виде суммы слагаемых, отвечающих вещественной и невещественной частям спектра оператор-функций, являющихся символами этих уравнений.
\end{abstract}

Ключевые слова: интегро-дифференциальное уравнение, оператор-функция, спектральный анализ.

\section{REPRESENTATION OF SOLUTIONS OF VOLTERRA INTEGRO-DIFFERENTIAL EQUATIONS WITH FRACTIONAL-EXPONENTIAL KERNELS}

\author{
(c) 2021 V. V. VLASOV, N. A. RAUTIAN
}

\begin{abstract}
In this paper, we study the asymptotic behavior of solutions of integro-differential equations on the basis of spectral analysis of their symbols. For this, we represent strong solutions of these equations as the sum of terms corresponding to the real and non-real parts of the spectrum of symbols of these equations.
\end{abstract}

Keywords and phrases: integro-differential equation, operator-function, spectral analysis.

AMS Subject Classification: 47G20, 34K30, 47A56, 34K12

1. Введение. Работа посвящена исследованию интегро-дифференциальных уравнений с неограниченными операторными коэффициентами в гильбертовом пространстве. Рассматриваемые уравнения представляют собой абстрактное гиперболическое уравнение, возмущенное слагаемыми, содержащими вольтерровы интегральные операторы. Эти уравнения могут быть реализованы как интегро-дифференциальные уравнения в частных производных, возникающие в теории вязкоупругости (см. [12,14]), а также как интегро-дифференциальные уравнения Гуртина-Пипкина (см. $[13,17,18])$, которые описывают процесс распространения тепла в средах с памятью с конечной скоростью, кроме того, указанные уравнения возникают в задачах усреднения в многофазных средах (закон Дарси) (см. $[1,11])$.

В предлагаемой статье установлено существование и единственность сильных решений начальных задач для упомянутых интегро-дифференциальных уравнений. На основании результатов о структуре и локализации спектра оператор-функций, являющихся символами указанных уравнений, получено представление сильных решений этих уравнений.

Работа выполнена при поддержке Российского фонда фундаментальных исследований (проект № 20-01-00288а). 
Спектр указанных оператор-функций содержит невещественную компоненту, состоящую из нормальных собственных значений конечной алгебраической кратности.

В статье приведена асимптотика невещественных собственных значений символов указанных интегро-дифференциальных уравнений. На этом основании получены оценки компоненты решения, отвечающей невещественной части спектра символа указанных интегро-дифференциальных уравнений, а также получены оценки второй компоненты решения, отвечающей поведению символа на разрезе отрицательной вещественной полуоси.

Наш подход к исследованию интегро-дифференциальных уравнений основан на спектральном анализе оператор-функций и существенно отличается от подходов, приведенных в известной нам литературе, посвященной, в основном, результатам о корректной разрешимости и оценке решений. Таким образом, мы получаем не только результаты о корректной разрешимости, но и представление решений в виде рядов, отражающих структуру решений, соответствующую структуре спектра символов рассматриваемых интегро-дифференциальных уравнений. Тем самым нам удается получить более полную информацию о поведении решений.

2. Определения и обозначения. Пусть $H$-сепарабельное гильбертово пространство, $A-$ самосопряженный положительный оператор, $A^{*}=A$, действующий в пространстве $H$, имеющий компактный обратный. Рассмотрим задачу

$$
\begin{gathered}
\frac{d^{2} u}{d t^{2}}+A^{2} u-\int_{0}^{t} K(t-s) A^{2} u(s) d s=f(t), \quad t \in \mathbb{R}_{+}, \\
u(+0)=\varphi_{0}, \quad u^{(1)}(+0)=\varphi_{1} .
\end{gathered}
$$

Скалярная функция $K(t)$ имеет представление

$$
K(t)=\sum_{j=1}^{\infty} c_{j} R_{j}(t)
$$

где $c_{j}>0, j \in \mathbb{N}$, функции $R_{j}(t)$ - дробно-экспоненицальные функции (см. [14, гл. I]), которые имеют вид

$$
R_{j}(t)=t^{\alpha-1} \sum_{n=0}^{\infty} \frac{\left(-\beta_{j}\right)^{n} t^{n \alpha}}{\Gamma[(n+1) \alpha]}, \quad 0<\alpha \leqslant 1,
$$

$\Gamma(\cdot)$ - гамма-функция Эйлера. При этом предполагается, что последовательность $\left\{\beta_{j}\right\}$ удовлетворяет следующим условиям: $0<\beta_{j}<\beta_{j+1}, j \in \mathbb{N}, \beta_{j} \rightarrow+\infty, j \rightarrow+\infty$. Кроме того, выполнены условия

$$
\sum_{j=1}^{\infty} \frac{c_{j}}{\beta_{j}}<1
$$

Преобразование Лапласа функции $R_{j}(t)$ имеет вид

$$
\hat{R}_{j}(\lambda)=\frac{1}{\lambda^{\alpha}+\beta_{j}}
$$

(см. [14, гл. I]). При этом под $\lambda^{\alpha}(0<\alpha \leqslant 1)$ понимается главная ветвь многозначной функции $f(\lambda)=\lambda^{\alpha}, \lambda \in \mathbb{C}$ с разрезом по отрицательной действительной полуоси $\lambda^{\alpha}=\left|\lambda^{\alpha}\right| e^{i \alpha \arg \lambda}$, $-\pi<\arg \lambda<\pi$. Применяя обратное преобразование Лапласа к главной ветви функции $\hat{R}_{j}(\lambda)$, получено (см. $\left[14\right.$, гл. I]) следующее интегральное представление функции $R_{j}(t)$

$$
R_{j}(t)=\frac{1}{2 \pi i} \lim _{R \rightarrow+\infty} \int_{\gamma-i R}^{\gamma+i R} \frac{e^{\lambda t} d \lambda}{\lambda^{\alpha}+\beta_{j}}=\frac{\sin \pi \alpha}{\pi} \int_{0}^{+\infty} \frac{e^{-t \tau} d \tau}{\tau^{\alpha}+2 \beta_{j} \cos \pi \alpha+\beta_{j}^{2} \tau^{-\alpha}} .
$$

Рассматривая преобразование Лапласа уравнения (1) при однородных начальных условиях, получаем уравнение $L(\lambda) \hat{u}(\lambda)=\hat{f}(\lambda)$, где оператор-функция

$$
L(\lambda)=\lambda^{2} I+A^{2}-\hat{K}(\lambda) A^{2}
$$


является символом этого уравнения, а $\hat{u}(\lambda)$ и $\hat{f}(\lambda)$ - преобразования Лапласа вектор-функций $u(t)$ и $f(t)$, соответственно. Здесь $\hat{K}(\lambda)$ - преобразование Лапласа ядра $K(t)$, имеющее представление

$$
\hat{K}(\lambda)=\sum_{j=1}^{\infty} \frac{c_{j}}{\lambda^{\alpha}+\beta_{j}}, \quad 0<\alpha \leqslant 1
$$

В предлагаемой работе мы получаем результат о представлении сильных решений уравнения (1) в весовых пространствах Соболева на положительной полуоси на основе спектрального анализа оператор-функции $L(\lambda)$, являющейся символом указанного уравнения.

В наших предшествующих работах $[2-5,22,23]$ проводилось подробное исследование задачи (1)(2) в случае, когда ядро $K(t)$ было представимо рядом убывающих экспонент с положительными коэффициентами, что равносильно случаю $\alpha=1$ в представлении (3). Наш подход к исследованию основывался на спектральном анализе оператор-функции (6), который также дает возможность получить результат о корректной разрешимости и представление решения указанной задачи в виде ряда по экспонентам, соответствующим точкам спектра оператор-функции $L(\lambda)$. Отметим также, что результаты работ [2-4,23] подытожены в главе 3 монографии [5].

\section{3. Формулировка результатов.}

3.1. Корректная разрешимость. Превратим область определения $\operatorname{Dom}\left(A^{\beta}\right)$ оператора $A^{\beta}$, $\beta>0$, в гильбертово пространство $H_{\beta}$, введя на $\operatorname{Dom}\left(A^{\beta}\right)$ норму $\|\cdot\|_{\beta}=\left\|A^{\beta} \cdot\right\|$, эквивалентную норме графика оператора $A^{\beta}$.

Через $W_{2, \gamma}^{n}\left(\mathbb{R}_{+}, A^{n}\right)$ обозначим пространство Соболева вектор-функций на полуоси $\mathbb{R}_{+}=(0, \infty)$ со значениями в $H$, снабженное нормой

$$
\|u\|_{W_{2, \gamma}^{n}\left(\mathbb{R}_{+}, A^{n}\right)} \equiv\left(\int_{0}^{\infty} e^{-2 \gamma t}\left(\left\|u^{(n)}(t)\right\|_{H}^{2}+\left\|A^{n} u(t)\right\|_{H}^{2}\right) d t\right)^{1 / 2}, \quad \gamma \geqslant 0 .
$$

Подробнее о пространствах $W_{2, \gamma}^{n}\left(\mathbb{R}_{+}, A^{2}\right)$ см. монографию [20, глава 1]. Для $n=0$ полагаем $W_{2, \gamma}^{0}\left(\mathbb{R}_{+}, A^{0}\right)=L_{2, \gamma}\left(\mathbb{R}_{+}, H\right)$, где $L_{2, \gamma}\left(\mathbb{R}_{+}, H\right)$ обозначено пространство измеримых функций со значениями в пространстве $H$, снабженное нормой

$$
\|f\|_{L_{2, \gamma}\left(\mathbb{R}_{+}, H\right)}=\left(\int_{0}^{+\infty} e^{-2 \gamma t}\|f(t)\|_{H}^{2} d t\right)^{1 / 2} .
$$

Определение. Будем называть вектор-функцию $u$ сильным решением задачи (1)-(2), если она принадлежит пространству $W_{2, \gamma}^{2}\left(\mathbb{R}_{+}, A^{2}\right)$ для некоторого $\gamma \geqslant 0$, удовлетворяет уравнению $(1)$ почти всюду на полуоси $\mathbb{R}_{+}$и начальным условиям $(2)$.

Теорема 1. Предположим, что вектор-функция $A f(t) \in L_{2, \gamma_{0}}\left(\mathbb{R}_{+}, H\right)$ для некоторого $\gamma_{0}>0$, ядро $K(t)$ представимо в виде (3), (4) с постоянной $\alpha(0<\alpha<1)$, а также выполняется условие (5), кроме того, $\varphi_{0} \in H_{3}, \varphi_{1} \in H_{2}$. Тогда существует такое $\gamma_{1}>\gamma_{0}$, что для всех $\gamma \geqslant \gamma_{1}$ задача (1)-(2) имеет единственное сильное решение в пространстве $W_{2, \gamma}^{2}\left(\mathbb{R}_{+}, A^{2}\right)$, удовлетворяющее неравенству

$$
\|u\|_{W_{2, \gamma}^{2}\left(\mathbb{R}_{+}, A^{2}\right)} \leqslant d\left(\|A f\|_{L_{2, \gamma}\left(\mathbb{R}_{+}, H\right)}+\left\|A^{3} \varphi_{0}\right\|_{H}+\left\|A^{2} \varphi_{1}\right\|_{H}\right),
$$

с постоянной $d$, не зависящей от вектор-функции $f$ и векторов $\varphi_{0}, \varphi_{1}$.

Доказательство теоремы 1 изложено в [8]. Следует отметить, что метод, используемый нами для доказательства корректной разрешимости начальных задач для абстрактных интегродифференциальных уравнений, существенно отличается от более традиционного подхода, использованного Л. Пандолфи в [20], где разрешимость изучается в функциональных пространствах на конечном временном интервале $(0, T)$. В нашей работе разрешимость изучается в весовых пространствах Соболева $W_{2, \gamma}^{2}\left(\mathbb{R}_{+}, A\right)$ вектор-функций на положительной полуоси $\mathbb{R}_{+}$, где $A$ - положительный самосопряженный оператор в гильбертовом пространстве. Доказательство 
нашей теоремы 1 о разрешимости существенно использует гильбертову структуру пространств $W_{2, \gamma}^{2}\left(\mathbb{R}_{+}, A\right), L_{2, \gamma}\left(\mathbb{R}_{+}, H\right)$, а также теорему Пэли-Винера.

Следует отметить, что в настоящее время имеется обширная литература, посвященная изучению интегро-дифференциальных уравнений с неограниченными операторными коэффициентами в банаховых и гильбертовых пространствах. Ограничимся здесь упоминанием статей $[16,20,24]$, а также монографий $[15,21]$. Особое внимание хотелось бы обратить на монографию [15], как наиболее близкую к тематике настоящей статьи (см. также приведенную в ней библиографию).

3.2. Спектральный анализ. Занумеруем собственные значения $a_{j}$ оператора $A\left(A e_{j}=a_{j} e_{j}\right)$ в порядке возрастания: $0<a_{1}<a_{2}<\ldots<a_{n}<\ldots, a_{n} \rightarrow+\infty$ при $n \rightarrow+\infty$. Соответствующие собственные векторы $\left\{e_{j}\right\}_{j=1}^{\infty}$ образуют ортонормированный базис пространства $H$. Рассмотрим сужение оператор-функции $L(\lambda)$ на одномерное подпространство, натянутое на вектор $e_{n}$ :

$$
l_{n}(\lambda)=\left(L(\lambda) e_{n}, e_{n}\right)=\lambda^{2}+a_{n}^{2}\left(1-\sum_{k=1}^{\infty} \frac{c_{k}}{\lambda^{\alpha}+\beta_{k}}\right) .
$$

Перейдем к изучению структуры спектра оператор-функции $L(\lambda)$ в случае, когда выполнено условие (5).

Теорема 2. Пусть выполнено условие (5). Тогда спектр оператор-функиии $L(\lambda)$ лежит в открытой левой полуплоскости.

Таким образом, условие (5) обеспечивает устойчивость задачи (1)-(2).

Теорема 3. Пусть выполнены условия (5) $и c_{j}=0$ для всех $j$, больших некоторого $N \in \mathbb{N}$. Тогда спектр оператор-функиии $L(\lambda)$ представим в виде

$$
\sigma(L):=\overline{\left\{\lambda_{n}^{ \pm} \in \mathbb{C} \backslash \mathbb{R}, \lambda_{n}^{-}=\overline{\lambda_{n}^{+}} \mid n \in \mathbb{N}\right\}}
$$

где $\lambda_{n}^{ \pm}$-невещественные собственнье значения оператор-функиии $L(\lambda)$, которье для каждого достаточно большого $n \in \mathbb{N}$ имегот асимптотику

$$
\lambda_{n}^{ \pm}=-\sin \left(\frac{\pi \alpha}{2}\right) a_{n}^{1-\alpha} \frac{Q}{2} \pm i a_{n}\left(1-\cos \left(\frac{\pi \alpha}{2}\right) a_{n}^{-\alpha} \frac{Q}{2}\right)+o\left(a_{n}^{1-\alpha}\right), \quad n \rightarrow+\infty
$$

здесъ

$$
Q=\sum_{j=1}^{N} c_{j}
$$

Замечание. При $\alpha=1$ асимптотическая формула (11) переходит в ранее известную асимптотическую формулу (2.15) из [22] (см. также [5]).

Полные доказательства теорем 2, 3 приведены в [6]; доказательства близких результатов имеются в $[7-9,15,16,21,24]$.

Отметим, что оператор-функция вида (6) в случае, когда ядра интегральных операторов являются рядами убывающих экспонент с положительными коэффициентами, изучалась в [3, 22]. Теоремы 2, 3 представляют собой естественное развитие результатов работ [3,22].

Замечание (см. [6, замечание 3.1]). При нарушении условия (5), т.е. при

$$
\sum_{j=1}^{\infty} \frac{c_{j}}{\beta_{j}}>1
$$

в правой полуплоскости имеется бесконечное число невещественных собственных значений оператор-функции $L(\lambda)$. При выполнении условия

$$
\sum_{j=1}^{\infty} \frac{c_{j}}{\gamma_{j}}=1
$$

точка $\lambda=0$ принадлежит спектру оператор-функции $L(\lambda)$ и является собственным значением бесконечной кратности. 
3.3. Представление решений. Сформулируем результат о представлении сильного решения задачи (1)-(2). Введем следующие обозначения:

$$
\begin{gathered}
\mathcal{K}_{n}(\tau)=\frac{a_{n}^{2}\left(\hat{K}_{-}(-\tau)-\hat{K}_{+}(-\tau)\right)}{\left(\tau^{2}+a_{n}^{2}\left(1-\hat{K}_{+}(-\tau)\right)\right)\left(\tau^{2}+a_{n}^{2}\left(1-\hat{K}_{-}(-\tau)\right)\right)} \\
\hat{K}_{ \pm}(-\tau)=\sum_{k=1}^{n} \frac{c_{k}}{\tau^{\alpha} e^{ \pm i \pi \alpha}+\beta_{k}}
\end{gathered}
$$

Теорема 4. Пусть выполнены условия теоремы $3, \alpha \in(0,1 / 2), f(t) \equiv 0$. Тогда сильное решение задачи (1)-(2) представимо в виде

$$
u(t)=u_{I}(t)+u_{R}(t), \quad t>0,
$$

где вектор-функиия и I $(t)$ представима в виде

$$
u_{I}(t)=\sum_{n=1}^{\infty}\left(\omega_{n}\left(t, \lambda_{n}^{+}\right)+\omega_{n}\left(t, \lambda_{n}^{-}\right)\right) e_{n}, \quad \omega_{n}(t, \lambda)=\frac{\left(\varphi_{1 n}+\lambda \varphi_{0 n}\right) e^{\lambda t}}{l_{n}^{(1)}(\lambda)},
$$

а вектор-функиця и $u_{R}(t)-$ в виде

$$
u_{R}(t)=\sum_{n=1}^{\infty} u_{R n}(t) e_{n}, \quad u_{R n}(t)=\int_{0}^{\infty} e^{-t \tau} \mathcal{K}_{n}(\tau)\left(-\tau \varphi_{0 n}+\varphi_{1 n}\right) d \tau
$$

при этом ряды (13), (14) сходятся по норме пространства $H$ и $\lambda_{n}^{ \pm}-$невещественные собственные значения оператор-функции $L(\lambda), \varphi_{k n}=\left(\varphi_{k}, e_{n}\right), n \in \mathbb{N}, k=1,2$.

В нижеследующих теоремах 5 и 8 приведены оценки вектор-функций $u_{I}(t)$ и $u_{R}(t)$. Отметим, что компонента $u_{I}(t)$ соответствует невещественным собственным значениям $\lambda_{n}^{ \pm}$и отвечает за волновой характер поведения решений. Компонента $u_{R}(t)$ отвечает за поведение оператор-функции $L^{-1}(\lambda)$ на разрезе отрицательной полуоси.

Обозначим через $P_{n}$ ортопроектор на подпространство, являющееся линейной оболочкой векторов $\left\{e_{j}\right\}_{j=1}^{n}$, а через $Q_{n}$ ортопроектор на подпространство, ортогональное подпространству $P_{n} H$ т.е. $Q_{n}=I-P_{n}$ и пространство $H$ представимо в виде ортогональной суммы

$$
H=P_{n} H \oplus Q_{n} H .
$$

Приведем результаты об оценке проекций вектор-функции $u_{I}(t)$ на подпространства $Q_{n} H$ и $P_{n} H$.

Теорема 5. Пусть выполнены условия теоремы \& и начальные данные $\varphi_{0} \in H_{3}, \varphi_{1} \in H_{2}$. Тогда для любого $\varepsilon>0$ существуют такое натуральное число $n_{0}$, что для вектор-функиии $u_{I}(t)$, определенной соотношением (13), выполнены следующие оценки

$$
\begin{gathered}
\left\|Q_{n_{0}} A^{m} u_{I}(t)\right\| \leqslant \theta_{1}\left\|Q_{n_{0}} e^{-k A^{1-\alpha} t} A^{m} \varphi_{0}\right\|+\theta_{2}\left\|Q_{n_{0}} e^{-k A^{1-\alpha} t} A^{m-1} \varphi_{1}\right\|, \quad t>0, \\
0<k=\frac{1}{2} \sin \left(\frac{\pi \alpha}{2}\right) \sum_{j=1}^{N} c_{j}-\varepsilon \\
\left\|P_{n_{0}} A^{m} u_{I}(t)\right\| \leqslant \theta_{3} e^{-\delta t}\left\{\left\|P_{n_{0}} A^{m} \varphi_{0}\right\|+\left\|P_{n_{0}} A^{m-1} \varphi_{1}\right\|\right\}, \quad t>0 \\
\delta=\operatorname{dist}\left(\left\{\lambda_{j}^{ \pm}\right\}_{j=1}^{n_{0}},\{i y \mid y \in \mathbb{R}\}\right),
\end{gathered}
$$

где $m=0,1,2$, положительные постоянные $\theta_{1}, \theta_{2}, \theta_{3}$ не зависят от векторов $\varphi_{0}, \varphi_{1}$.

Заметим, что $\delta$ представляет собой расстояние от подмножества невещественных собственных значений $\left\{\lambda_{j}^{ \pm}\right\}_{j=1}^{n_{0}}$ до мнимой оси. Согласно теореме 2 спектр оператор-функции $L(\lambda)$ лежит в левой полуплоскости. 
Теорема 6. Пусть выполнены условия теоремы 4. Тогда для любого $\varepsilon>0$ вектор-функиия $w(t)$, определяемая соотношением (14), допускает следуюшую оценку:

$$
\begin{aligned}
\left\|A^{m} u_{R}(t)(t)\right\|^{2} \leqslant e^{-2 \varepsilon t}\left\{k_{1}\left\|A^{m-\alpha} \varphi_{0}\right\|^{2}\right. & \left.+k_{2}\left\|A^{m-1-\alpha} \varphi_{1}\right\|^{2}\right\}+ \\
& +k_{3}\left\{\varepsilon^{2(2+\alpha)}\left\|A^{m-2} \varphi_{0}\right\|^{2}+\varepsilon^{2(1+\alpha)}\left\|A^{m-2} \varphi_{1}\right\|^{2}\right\}, \quad t>0
\end{aligned}
$$

где $m=0,1,2$, положительные постоянные $k_{1}, k_{2}, k_{3}$ не зависят от векторов $\varphi_{0}, \varphi_{1}$.

Заметим также, что в формулировках теорем 5 и 6 не предполагается, что вектор-функция $u$ является решением задачи (1)-(2), т.е. разложение (13) носит абстрактный характер. Тем не менее, при выполнении условий теоремы 4 оценки (15), (17) можно применить к сильному решению задачи (1)-(2). Полные доказательства теорем 4-6 приведены в [10].

Теорема 7. Пусть выполнены условия теоремы $3, \alpha \in(0,1 / 2), \varphi_{0}=\varphi_{1} \equiv 0$. Тогда сильное решение задачи (1)-(2) представимо в виде

$$
u(t)=u_{I}(t)+u_{R}(t), \quad t>0,
$$

где вектор-функиия и $u_{I}(t)$ представима в виде

$$
\begin{gathered}
u_{I}(t)=\sum_{n=1}^{\infty}\left(\omega_{n}\left(t, \lambda_{n}^{+}\right)+\omega_{n}\left(t, \lambda_{n}^{-}\right)\right) e_{n}, \\
\omega_{n}(t, \lambda)=\frac{1}{l_{n}^{(1)}(\lambda)} \int_{0}^{t} e^{\lambda(t-\tau)} f_{n}(\tau) d \tau, \quad f_{n}(\tau)=\left(f(\tau), e_{n}\right),
\end{gathered}
$$

а вектор-функция и $u_{R}(t)$ представима в виде

$$
u_{R}(t)=\sum_{n=1}^{\infty} u_{R n}(t) e_{n}, \quad u_{R n}(t)=\int_{0}^{t}\left(\int_{0}^{\infty} e^{-(t-\tau) \nu} \mathcal{K}_{n}(\nu) d \nu\right) f_{n}(\tau) d \tau,
$$

при этом ряды (13), (14) сходятся по норме пространства $Н$ и $\lambda_{n}^{ \pm}$- невещественные собственные значения оператор-функции $L(\lambda), \varphi_{k n}=\left(\varphi_{k}, e_{n}\right), n \in \mathbb{N}, k=1,2$.

Теорема 8. Пусть выполнены условия теоремы 7 и $A f(t) \in L_{2, \gamma_{0}}\left(\mathbb{R}_{+}, H\right)$ для некоторого $\gamma_{0}>0$. Тогда для любого $\varepsilon>0$ существуют такое натуральное число $n_{0}$, что для решения задачи (1)-(2) справедлива следующая оценка:

$$
\begin{aligned}
\left\|A^{m} u(t)\right\| \leqslant & d_{1} t \int_{0}^{t}\left\|Q_{n_{0}} e^{-k A^{1-\alpha}}(t-\tau) A^{m-1} f(\tau)\right\|^{2} d \tau+d_{2} t \int_{0}^{t} e^{-2 \delta(t-\tau)}\left\|P_{n_{0}} A^{m-1} f(\tau)\right\|^{2} d \tau+ \\
& +t\left\{k_{1} \int_{0}^{t} e^{-2 \varepsilon(t-\tau)}\left\|A^{m-(1+\alpha)} f(\tau)\right\|^{2} d \tau+k_{2} \varepsilon^{2(\alpha+1)} \int_{0}^{t}\left\|A^{m-2} f(\tau)\right\|^{2} d \tau\right\}, \quad t>0
\end{aligned}
$$

где $m=0,1,2$, положительные постоянные $d_{1}, d_{2}, k_{1}, k_{2}$ не зависят от вектор-функиии $f(t)$, константы $k$ и бопределяются формулами (16) и (18), соответственно.

В случае реализации оператора $A$ как самосопряженного дифференциального оператора в частных производных по пространственным переменным оценки (15), (17), (19), (23) можно рассматривать как оценки решения $u$ соответствующей начально-краевой задачи для интегродифференциального уравнения в частных производных. При этом умножению на степени оператора $A$ соответствует применение дифференциальных операторов по пространственным переменным. 


\section{4. Доказательства основных утверждений.}

4.1. Доказательство теоремы 7. Рассмотрим задачу (1)-(2) при условии, что $\varphi_{0}=\varphi_{1}=0$. По условию теоремы 1 , задача (1)-(2) имеет единственное решение $u(t) \in W_{2, \gamma}^{2}\left(\mathbb{R}_{+}, A^{2}\right), \gamma>0$, которое можно представить в виде

$$
u(t)=\int_{0}^{t} v(t, \tau) d \tau
$$

где вектор-функция $v(t, \tau)$ является решением следующей задачи:

$$
\begin{gathered}
\frac{d^{2} v(t, \tau)}{d t^{2}}+A^{2} v(t, \tau)-\int_{\tau}^{t} K(t-s) A^{2} v(s, \tau) d s=0, \quad t>\tau, \\
v(\tau, \tau)=0, \quad v_{t}^{\prime}(\tau, \tau)=f(\tau), \quad \tau>0,
\end{gathered}
$$

$v(t, \tau)=0$ при $t<\tau$. Действительно, поскольку $v(t, \tau) \in W_{2, \gamma}^{2}\left(\mathbb{R}_{+}, A^{2}\right)$, то

$$
A^{2} u(t)=\int_{0}^{t} A^{2} v(t, \tau) d \tau
$$

Продифференцируем решение $u(t)$ по переменной $t$, учитывая условия $(25)$ :

$$
\begin{gathered}
u_{t}(t)=v(t, t)+\int_{0}^{t} v_{t}(t, \tau) d \tau=\int_{0}^{t} v_{t}(t, \tau) d \tau, \\
u_{t t}(t)=v_{t}(t, t)+\int_{0}^{t} v_{t t}(t, \tau) d \tau=f(t)+\int_{0}^{t} v_{t t}(t, \tau) d \tau .
\end{gathered}
$$

Меняя порядок интегрирования, получаем

$$
\int_{0}^{t} K(t-s) A^{2}\left(\int_{0}^{s} v(s, \tau) d \tau\right) d s=\int_{0}^{t}\left(\int_{\tau}^{t} K(t-s) A^{2} v(s, \tau) d s\right) d \tau .
$$

Возможность дифференцирования и замены переменных обеспечивается тем, что вектор-функция $v(t, \tau)$ по переменной $t$ принадлежит пространству $W_{2, \gamma}^{2}\left(\mathbb{R}_{+}, A^{2}\right)$, а ядро $K(t)$ принадлежит пространству $L_{1}\left(\mathbb{R}_{+}\right)$.

Преобразование Лапласа по переменной $t$ сильного решения задачи $(24),(25)$ имеет вид

$$
\hat{v}(\lambda, \tau)=L^{-1} f(\tau) e^{-\lambda \tau}
$$

где оператор-функция $L(\lambda)$ является символом уравнения (24) и имеет вид (6). Следовательно,

$$
\hat{v}_{n}(\lambda, \tau)=\frac{f_{n}(\tau) e^{-\lambda \tau}}{l_{n}(\lambda)} .
$$

Спроектируем $\hat{v}(\lambda, \tau)$ на подпространство, натянутое на вектор $e_{n}$ :

$$
\left(\hat{v}(\lambda, \tau), e_{n}\right)=\hat{v}_{n}(\lambda, \tau)=l_{n}^{-1}(\lambda) f_{n}(\tau) e^{-\lambda \tau}
$$

Согласно формуле обращения преобразования Лапласа, получаем

$$
v_{n}(t, \tau)=\frac{v \cdot p .}{2 \pi i} \int_{\gamma-i \infty}^{\gamma+i \infty} l_{n}^{-1}(\lambda) f_{n}(\tau) e^{\lambda(t-\tau)} d \lambda
$$


Согласно теореме 3 функция $\hat{v}_{n}(\lambda, \tau)$ имеет полюсы в точках $\lambda_{n}^{ \pm}$комплексной плоскости, с разрезом по отрицательной действительной полуоси. В комплексной плоскости рассмотрим контур Г следующего вида:

$$
\Gamma=\Gamma_{0} \cup \Gamma_{1} \cup C_{R}^{+} \cup R^{+} \cup R^{-} \cup C_{R}^{-} \cup \Gamma_{2},
$$

где

$$
\begin{gathered}
\Gamma_{0}=\{\lambda: \operatorname{Re} \lambda=\gamma,-R \leqslant \operatorname{Im} \lambda \leqslant R\}, \quad \Gamma_{1}=\{\lambda: 0 \leqslant \operatorname{Re} \lambda \leqslant \gamma, \operatorname{Im} \lambda=R\}, \\
C_{R}^{+}=\left\{\lambda: \lambda=\operatorname{Re}^{i \varphi}, \frac{\pi}{2} \leqslant \varphi \leqslant \pi\right\}, \quad R^{+}=\{\lambda: \operatorname{Im} \lambda=0,-R \leqslant \operatorname{Re} \lambda \leqslant 0\}, \\
R^{-}=\{\lambda: \operatorname{Im} \lambda=0,-R \leqslant \operatorname{Re} \lambda \leqslant 0\}, \quad C_{R}^{-}=\left\{\lambda: \lambda=\operatorname{Re}^{i \varphi},-\pi \leqslant \varphi \leqslant \frac{-3}{2} \pi\right\}, \\
\Gamma_{2}=\{\lambda: 0 \leqslant \operatorname{Re} \lambda \leqslant \gamma, \operatorname{Im} \lambda=-R\} .
\end{gathered}
$$

Предполагается, что контур Г обходится против часовой стрелки. Напомним (см. доказательство леммы 3.1 из [6]), что в плоскости с разрезом по отрицательной полуоси справедлива следующая оценка

$$
|\hat{K}(\lambda)| \leqslant \frac{\text { const }}{|\lambda|^{\alpha}}
$$

Для достаточно больших по модулю $\lambda$ в плоскости с разрезом по отрицательной полуоси имеем:

$$
\left|\frac{\lambda}{\lambda^{2}+a_{n}^{2}(1-\hat{K}(\lambda))}\right| \leqslant \frac{|\lambda|}{|\lambda|^{2}\left|1+a_{n}^{2}\left(\frac{1}{\lambda^{2}}-\frac{\hat{K}(\lambda)}{\lambda^{2}}\right)\right|} \leqslant \frac{1}{|\lambda||1-| \frac{a_{n}^{2}}{\lambda^{2}}+\frac{a_{n}^{2} \hat{K}(\lambda)}{\lambda^{2}}||} .
$$

Заметим, что при фиксированном $a_{n}$ и при достаточно больших по модулю $\lambda=\operatorname{Re}^{i \varphi}$ справедлива оценка

$$
\left|\frac{a_{n}^{2}}{R^{2} e^{2 i \varphi}}+\frac{a_{n}^{2}}{R^{(2+\alpha)} e^{i \varphi(2+\alpha)}}\right| \leqslant \frac{2 a_{n}^{2}}{R^{2}} .
$$

Поэтому, начиная с некоторого $R_{0}>2 a_{n}$, при $R>R_{0}$ справедливо неравенство

$$
\frac{2 a_{n}^{2}}{R^{2}}<\frac{1}{2}
$$

Отсюда из оценок (27)-(30) вытекает, что

$$
\left|l_{n}^{-1}(\lambda) f_{n}(\tau)\right| \leqslant \frac{2}{R^{2}}\left|f_{n}(\tau)\right|
$$

при $R>2 a_{n}, \lambda=\operatorname{Re}^{i \varphi}$. Из оценки (31) и леммы Жордана следует, что при $t>0$

$$
\int_{C_{R}^{ \pm}} l_{n}^{-1}(\lambda) f_{n}(\tau) e^{\lambda(t-\tau)} d \lambda \rightarrow 0, \quad|\lambda|=R \rightarrow+\infty
$$

Покажем теперь, что интегралы от функции $l_{n}^{-1}(\lambda) f_{n}(\tau) e^{\lambda(t-\tau)}, t>\tau$, по отрезкам $\Gamma_{1}$ и $\Gamma_{2}$ стремятся к нулю при $R \rightarrow+\infty$. В самом деле, в соответствии с (31) получаем следующую цепочку неравенств

$$
\left|\int_{ \pm i R}^{ \pm i R+\gamma} l_{n}^{-1}(\lambda) f_{n}(\tau) e^{\lambda(t-\tau)} d \lambda\right| \leqslant \int_{0}^{\gamma}\left|l_{n}^{-1}(x \pm i R)\right|\left|f_{n}(\tau)\right| e^{x(t-\tau)} d x \leqslant \leqslant \frac{2}{R^{2}}\left|f_{n}(\tau)\right| \frac{e^{\gamma(t-\tau)}-1}{t-\tau}
$$

Легко видеть, что правая часть (32) стремится к нулю при $R \rightarrow+\infty$.

Выясним поведение $\hat{v}_{n}(\lambda, \tau)$ на верхнем и на нижнем берегах разреза соответственно. Вначале рассмотрим верхний берег разреза от $-R$ до $-\varepsilon$. На верхнем берегу разреза получаем выражение, 
имеющее следующий вид при $\lambda=x$ :

$$
\int_{-R}^{-\varepsilon_{-}^{-}} \frac{e^{x(t-\tau)} f_{n}(\tau)}{x^{2}+a_{n}^{2}\left(1-\hat{K}_{+}(x)\right)} d x=\int_{\varepsilon}^{R} \frac{e^{-\nu(t-\tau)} f_{n}(\tau)}{\nu^{2}+a_{n}^{2}\left(1-\hat{K}_{+}(-\nu)\right)} d \nu,
$$

где функция $\hat{K}_{+}(-\nu)$ имеет вид

$$
\hat{K}_{+}(-\nu)=\sum_{k=1}^{n} \frac{c_{k}}{\nu^{\alpha} e^{i \pi \alpha}+\beta_{k}} .
$$

Рассмотрим теперь возможность предельного перехода при $\varepsilon \rightarrow+0$ и $R \rightarrow+\infty$. Поскольку по условию теоремы

$$
\sum_{k=1}^{n} \frac{c_{k}}{\beta_{k}}<1
$$

то подынтегральное выражение в формуле (33) не будет иметь особенностей в окрестности начала координат, т.е. интеграл будет собственным при $\tau \rightarrow+0$, и в интеграле (33) можно положить $\varepsilon=0$. В силу экспоненциального убывания подынтегральной функции при $\tau \rightarrow+\infty$ в интеграле можно перейти к пределу при $R \rightarrow+\infty$. Таким образом, на верхнем берегу разреза после предельного перехода получаем выражение

$$
\int_{0}^{+\infty} \frac{e^{-\nu(t-\tau)} f_{n}(\tau)}{\nu^{2}+a_{n}^{2}\left(1-\hat{K}_{+}(-\nu)\right)} d \nu
$$

Совершенно аналогично на нижнем берегу разреза в пределе получим выражение

$$
-\int_{0}^{+\infty} \frac{e^{-\nu(t-\tau)} f_{n}(\tau)}{\nu^{2}+a_{n}^{2}\left(1-\hat{K}_{-}(-\nu)\right)} d \nu, \quad \text { где } \quad \hat{K}_{-}(-\nu)=\sum_{k=1}^{n} \frac{c_{k}}{\nu^{\alpha} e^{-i \pi \alpha}+\beta_{k}} .
$$

Осуществим теперь предельный переход при интегрировании функции $\hat{v}_{n}(\lambda, \tau)$ по контуру $\Gamma$ при $R \rightarrow+\infty$. Тогда интеграл по контуру $\Gamma_{0}$ в пределе при $R \rightarrow+\infty$ даст функцию $u_{n}(t)$, по формуле обращения для преобразования Лапласа. Интегралы от функции $\hat{v}_{n}(\lambda, \tau)$ по контурам $\Gamma_{1}, \Gamma_{2}, C_{R}^{+}$, $C_{R}^{-}$будут стремиться к нулю по доказанному. В свою очередь, интегралы по разрезам в пределе при $R \rightarrow+\infty$ перейдут в интегралы вида (34), (35). Таким образом, переходя к пределу при $R \rightarrow+\infty$, получим, что функция $u_{n}(t)$ будет допускать следующее представление:

$$
v_{n}(t, \tau)=v_{n}^{+}(t, \tau)+v_{n}^{-}(t, \tau)+v_{R n}(t, \tau)
$$

где

$$
\begin{gathered}
v_{n}^{+}(t, \tau)=\operatorname{res}_{\lambda=\lambda_{n}^{+}}\left(\hat{v}_{n}(\lambda, \tau) e^{\lambda t}\right)=\frac{f_{n}(\tau) e^{\lambda_{n}^{+}(t-\tau)}}{2 \lambda_{n}^{+}-a_{n}^{2} \hat{K}^{(1)}\left(\lambda_{n}^{+}\right)}, \\
v_{n}^{-}(t, \tau)=\operatorname{res}_{\lambda=\lambda_{n}^{-}}\left(\hat{v}_{n}(\lambda, \tau) e^{\lambda t}\right)=\frac{f_{n}(\tau) e^{\lambda_{n}^{-}(t-\tau)}}{2 \lambda_{n}^{-}-a_{n}^{2} \hat{K}^{(1)}\left(\lambda_{n}^{-}\right)} \\
v_{R n}(t, \tau)=\int_{0}^{+\infty} \frac{e^{-\nu(t-\tau)} f_{n}(\tau)}{\nu^{2}+a_{n}^{2}\left(1-\hat{K}_{+}(-\nu)\right)} d \nu-\int_{0}^{+\infty} \frac{e^{-\nu(t-\tau)} f_{n}(\tau)}{\left.\nu^{2}+a_{n}^{2}\left(1-\hat{K}_{-}-\nu\right)\right)} d \nu= \\
=\int_{0}^{\infty} e^{-(t-\tau) \nu} \mathcal{K}_{n}(\nu) f_{n}(\tau) d \nu
\end{gathered}
$$




$$
\begin{gathered}
\mathcal{K}_{n}(\nu)=\frac{a_{n}^{2}\left(\hat{K}_{-}(-\nu)-\hat{K}_{+}(-\nu)\right)}{\left(\nu^{2}+a_{n}^{2}\left(1-\hat{K}_{+}(-\nu)\right)\right)\left(\nu^{2}+a_{n}^{2}\left(1-\hat{K}_{-}(-\nu)\right)\right)}, \\
\hat{K}_{-}(-\nu)-\hat{K}_{+}(-\nu)=\sin (\pi \alpha) \nu^{\alpha} \sum_{k=1}^{n} \frac{c_{k}}{\left(\nu^{\alpha} \cos (\pi \alpha)+\beta_{k}\right)^{2}+\nu^{2 \alpha} \sin ^{2}(\pi \alpha)} .
\end{gathered}
$$

Итак, пока на формальном уровне получаем, что решение исходной задачи допускает представление в виде суммы ряда

$$
v(t, \tau)=\sum_{n=1}^{\infty}\left(v_{n}^{+}(t, \tau)+v_{n}^{-}(t, \tau)+v_{R n}(t, \tau)\right) e_{n} .
$$

Доказательство сходимости рядов (13), (14) приведено в [10], а рядов (21), (22) - получено в процессе доказательства теоремы 8, при получении оценок сумм этих рядов.

\section{2. Доказательство теоремы 8. Вначале исследуем на сходимость ряд}

$$
v^{+}(t, \tau)+v^{-}(t, \tau)=\sum_{n=1}^{\infty}\left(v_{n}^{+}(t, \tau)+v_{n}^{-}(t, \tau)\right) e_{n} .
$$

Напомним, что собственные значения являются комплексно-сопряженными $\bar{\lambda}_{n}^{+}=\lambda_{n}^{-}$в силу того, что коэффициенты разложения функции Работнова являются вещественными. По этой же причине $\hat{\bar{K}}^{(1)}\left(\lambda_{n}^{+}\right)=\hat{K}^{(1)}\left(\lambda_{n}^{-}\right)$. В силу этого справедливы представления

$$
v_{n}^{+}(t, \tau)=\frac{e^{\lambda_{n}^{+}(t-\tau)}\left(2 \lambda_{n}^{-}-\hat{K}^{(1)}\left(\lambda_{n}^{-}\right)\right) f_{n}(\tau)}{\left|2 \lambda_{n}^{+}-a_{n}^{2} \hat{K}^{(1)}\left(\lambda_{n}^{+}\right)\right|^{2}}, \quad v_{n}^{-}(t, \tau)=\frac{e^{\lambda_{n}^{-}(t-\tau)}\left(2 \lambda_{n}^{+}-\hat{K}^{(1)}\left(\lambda_{n}^{+}\right)\right) f_{n}(\tau)}{\left|2 \lambda_{n}^{+}-a_{n}^{2} \hat{K}^{(1)}\left(\lambda_{n}^{+}\right)\right|^{2}} .
$$

Отсюда получаем

$$
v_{n}^{+}(t, \tau)+v_{n}^{-}(t, \tau)=\frac{\left(4 \operatorname{Re}\left(\lambda_{n}^{-} e^{\lambda_{n}^{+}(t-\tau)}\right)-2 \operatorname{Re}\left(e^{\lambda_{n}^{+}(t-\tau)} \hat{K}^{(1)}\left(\lambda_{n}^{-}\right)\right)\right) f_{n}(\tau)}{\left|2 \lambda_{n}^{+}-a_{n}^{2} \hat{K}^{(1)}\left(\lambda_{n}^{+}\right)\right|^{2}} .
$$

Заметим, что при доказательстве леммы 3.1 в [6] была установлена оценка

$$
\left|\hat{K}^{(1)}(\lambda)\right| \leqslant \frac{\text { const }}{|\lambda|^{\alpha+1}}
$$

в области $\Omega_{\pi-\delta}=\{\lambda:|\arg \lambda|<\pi-\delta, 0<\delta<\pi / 2\}$. Воспользуемся этой оценкой с целью оценить знаменатель в выражении (42). Дальнейшему изложению предпошлем следующее предложение.

Предложение 1. Найдутся такие положстельные постоянные $d_{1} u d_{2}$, что для всех $n$, начиная с некоторого $n_{0} \in \mathbb{N}$, будут выполняться неравенства

$$
d_{1} a_{n} \leqslant\left|\lambda_{n}^{ \pm}\right| \leqslant d_{2} a_{n}
$$

Данное предложение является непосредственным следствием асимптотической формулы (11). В самом деле, левая часть неравенства (44) вытекает из очевидного неравенства $\left|\operatorname{Im} \lambda_{n}^{ \pm}\right| \leqslant\left|\lambda_{n}^{ \pm}\right|$, а также того, что при $n>n_{0}$

$$
\left|\operatorname{Im} \lambda_{n}^{ \pm}\right| \geqslant \frac{1}{2} a_{n}\left(1-\cos \frac{\alpha \pi}{2} a_{n}^{-\alpha} \frac{Q}{2}\right) .
$$

Правая часть неравенства (44) вытекает из следующей цепочки неравенств, справедливой при $n>n_{0}$ :

$$
\left|\lambda_{n}^{ \pm}\right| \leqslant\left|\operatorname{Re} \lambda_{n}^{ \pm}\right|+\left|\operatorname{Im} \lambda_{n}^{ \pm}\right| \leqslant 2\left|\operatorname{Im} \lambda_{n}^{ \pm}\right| \leqslant 2 a_{n} .
$$

Таким образом, из неравенств $(43)$ и (44) получаем, что при $n>n_{0}$

$$
\left|\hat{K}^{(1)}\left(\lambda_{n}^{ \pm}\right)\right| \leqslant \frac{C_{1}}{a_{n}^{1+\alpha}},
$$

с некоторой положительной постоянной $C_{1}$. Из этой оценки и неравенства (44) получаем, что при $n>n_{0}$

$$
\left|2 \lambda_{n}^{ \pm}-a_{n}^{2} \hat{K}^{(1)}\left(\lambda_{n}^{ \pm}\right)\right| \geqslant\left|2 \lambda_{n}^{ \pm}\right|-\left|a_{n}^{2} \hat{K}^{(1)}\left(\lambda_{n}^{ \pm}\right)\right| \geqslant k_{1} a_{n}-k_{2} a_{n}^{1-\alpha} \geqslant C_{2} a_{n}
$$


с некоторыми положительными постоянными $k_{1}, k_{2}, C_{2}$. Оценим сверху числители в выражении (42), используя неравенство (44), а также асимптотическую формулу (11). Для любого $\varepsilon>0$ найдется такое число $n_{0} \in \mathbb{N}$, что при $n>n_{0}$ числители первого и второго слагаемого допускают, соответственно, следующие оценки

$$
\begin{array}{r}
\left|2 \operatorname{Re}\left(e^{\lambda_{n}^{+}(t-\tau)}\left(2 \lambda_{n}^{-}-\hat{K}^{(1)}\left(\lambda_{n}^{-}\right)\right)\right) f_{n}(\tau)\right| \leqslant C_{5} e^{-k a_{n}^{1-\alpha}(t-\tau)}\left(a_{n}-C_{6} a_{n}^{-1-\alpha}\right)\left|f_{n}(\tau)\right| \leqslant \\
\leqslant d_{2} e^{-k a_{n}^{1-\alpha}(t-\tau)} a_{n}\left|f_{n}(\tau)\right|
\end{array}
$$

с положительными постоянными $C_{5}, C_{6}, d_{2}$ где

$$
k=\frac{1}{2} \sin \left(\frac{\pi \alpha}{2}\right) \sum_{j=1}^{N} c_{j}-\varepsilon .
$$

Таким образом, из оценок (45), (46) вытекает, что

$$
\left|v_{n}^{+}(t, \tau)+v_{n}^{-}(t, \tau)\right| \leqslant d_{3} e^{-k a_{n}^{1-\alpha}(t-\tau)} a_{n}^{-1}\left|f_{n}(\tau)\right|,
$$

Из последнего неравенства для функций

$$
u_{n}^{+}(t)=\int_{0}^{t} v_{n}^{+}(t, \tau) d \tau, \quad u_{n}^{-}(t)=\int_{0}^{t} v_{n}^{-}(t, \tau) d \tau
$$

получаем следуюшие неравенства:

$$
\left|u_{n}^{+}(t)+u_{n}^{-}(t)\right| \leqslant \int_{0}^{t}\left|v_{n}^{+}(t, \tau)+v_{n}^{-}(t, \tau)\right| d \tau \leqslant d_{4} \int_{0}^{t} e^{-k a_{n}^{1-\alpha}(t-\tau)} a_{n}^{-1}\left|f_{n}(\tau)\right| d \tau,
$$

В свою очередь, из последнего неравенства и неравенства Коши-Буняковского получаем

$$
\begin{aligned}
\left|u_{n}^{+}(t)+u_{n}^{-}(t)\right|^{2} \leqslant\left(\int_{0}^{t}\left|v_{n}^{+}(t, \tau)+v_{n}^{-}(t, \tau)\right| d \tau\right)^{2} & \leqslant d_{5}\left(\int_{0}^{t} e^{-k a_{n}^{1-\alpha}(t-\tau)} a_{n}^{-1}\left|f_{n}(\tau)\right| d \tau\right)^{2} \leqslant \\
& \leqslant d_{5} t\left(\int_{0}^{t} e^{-2 k a_{n}^{1-\alpha}(t-\tau)} a_{n}^{-2}\left|f_{n}(\tau)\right|^{2} d \tau\right)
\end{aligned}
$$

Отсюда получаем

$$
\sum_{n=n_{0}+1}^{\infty}\left|u_{n}^{+}(t)+u_{n}^{-}(t)\right|^{2} \leqslant d_{5} t \sum_{n=n_{0}+1}^{\infty}\left(\int_{0}^{t} e^{-2 k a_{n}^{1-\alpha}(t-\tau)} a_{n}^{-2}\left|f_{n}(\tau)\right|^{2} d \tau\right) .
$$

В неравенствах (47)-(49) постоянные $d_{3}, d_{4}, d_{5}$ не зависят от $n$.

Неравенство (50) может быть переписано

$$
\left\|Q_{n_{0}}\left(u^{+}(t)+u^{-}(t)\right)\right\|^{2} \leqslant d_{1} t \int_{0}^{t}\left\|Q_{n_{0}} e^{-k A^{1-\alpha}(t-\tau)} A^{-1} f(\tau)\right\|^{2} d \tau
$$

Оценка (17) компоненты решения $P_{n_{0}} u_{I}(t)$ немедленно вытекает из утверждения теоремы 2 о том, что спектр оператор-функции $L(\lambda)$ лежит в открытой левой полуплоскости. Следовательно, существует такое $\delta>0$, что конечное множество собственных значений $\left\{\lambda_{j}^{ \pm}\right\}_{j=1}^{n_{0}}$ отделено от мнимой оси вертикальной полосой $\{\lambda:-\delta<\operatorname{Re}<0\}$. Дословно повторяя предшествующие рассуждения от оценке $\left\|Q_{n_{0}}\left(u^{+}(t)+u^{-}(t)\right)\right\|^{2}$, получаем, что

$$
\left\|P_{n_{0}}\left(v^{+}(t, \tau)+v^{-}(t, \tau)\right)\right\| \leqslant \theta_{3} e^{-\delta(t-\tau)}\left\|P_{n_{0}} A^{-1} f(\tau)\right\| .
$$

Отсюда и из неравенства Коши-Буняковского получаем цепочку неравенств 


$$
\begin{aligned}
\left\|P_{n_{0}}\left(u^{+}(t)+u^{-}(t)\right)\right\|^{2} & =\left\|\int_{0}^{t} P_{n_{0}}\left(v^{+}(t, \tau)+v^{-}(t, \tau)\right) d \tau\right\|^{2} \leqslant \\
\leqslant & \left(\int_{0}^{t}\left\|P_{n_{0}}\left(v^{+}(t, \tau)+v^{-}(t, \tau)\right)\right\| d \tau\right)^{2} \leqslant \theta_{3} t \int_{0}^{t} e^{-\delta(t-\tau)}\left\|P_{n_{0}} A^{-1} f(\tau)\right\|^{2} d \tau .
\end{aligned}
$$

На основании оценок (51), (52) получаем сходимость ряда (21).

Займемся теперь изучением сходимости ряда (22). Перейдем к оценке функций $v_{R n}(t, \tau)=$ $\left(v_{R}(t, \tau), e_{n}\right)$, заданных формулой $(37)$

$$
v_{R n}(t, \tau)=\int_{0}^{+\infty} \frac{e^{-(t-\tau) \nu} a_{n}^{2}\left(\hat{K}_{-}(-\nu)-\hat{K}_{+}(-\nu)\right) f_{n}(\tau)}{\left(\nu^{2}+a_{n}^{2}\left(1-\hat{K}_{+}(-\nu)\right)\right)\left(\nu^{2}+a_{n}^{2}\left(1-\hat{K}_{-}(-\nu)\right)\right)} d \nu .
$$

Заметим, что

$$
\hat{K}_{-}(-\nu)-\hat{K}_{+}(-\nu)=\sin (\pi \alpha) \nu^{\alpha} \sum_{k=1}^{n} \frac{c_{k}}{\left(\nu^{\alpha} \cos (\pi \alpha)+\beta_{k}\right)^{2}+\nu^{2 \alpha} \sin ^{2}(\pi \alpha)} .
$$

Следовательно, для любого $\varepsilon>0$ на полуоси $\nu \in(\varepsilon,+\infty)$ справедливо неравенство

$$
\hat{K}_{-}(-\nu)-\hat{K}_{+}(-\nu) \leqslant \frac{\kappa}{\nu^{\alpha}},
$$

с положительной постоянной $\kappa$.

Поскольку $\alpha \in(0,1 / 2)$, то $\cos (\alpha \pi) \geqslant 0$. Учитывая это обстоятельство, оценим снизу знаменатель в выражении (53). Из неравенств

$$
\begin{aligned}
\left|\nu^{2}+a_{n}^{2}\left(1-\hat{K}_{ \pm}(-\nu)\right)\right| \geqslant \nu^{2}+a_{n}^{2}\left(1-\operatorname{Re} \hat{K}_{ \pm}(-\nu)\right) & \\
1-\operatorname{Re} \hat{K}_{ \pm}(-\nu) & =1-\sum_{k=1}^{n} \frac{c_{k}\left(\nu^{\alpha} \cos (\alpha \pi)+\beta_{k}\right)}{\nu^{2 \alpha}+2 \beta_{k} \nu^{\alpha} \cos (\alpha \pi)+\beta_{k}^{2}} \geqslant \\
& \geqslant 1-\sum_{k=1}^{n} \frac{c_{k}\left(\nu^{\alpha} \cos (\alpha \pi)+\beta_{k}\right)}{\nu^{2 \alpha} \cos ^{2}(\alpha \pi)+2 \beta_{k} \nu^{\alpha} \cos (\alpha \pi)+\beta_{k}^{2}}= \\
& =1-\sum_{k=1}^{n} \frac{c_{k}}{\nu^{\alpha} \cos (\alpha \pi)+\beta_{k}} \geqslant 1-\sum_{k=1}^{n} \frac{c_{k}}{\beta_{k}}=\delta^{2}>0
\end{aligned}
$$

с постоянной $\delta>0$, получаем

$$
\left|\nu^{2}+a_{n}^{2}\left(1-\hat{K}_{ \pm}(-\nu)\right)\right| \geqslant \nu^{2}+\delta^{2} a_{n}^{2} .
$$

Отсюда на основании (56), (57) приходим к неравенству

$$
\left|u_{R n}(t, \tau)\right| \leqslant \int_{0}^{\infty} e^{-\nu(t-\tau)} \frac{a_{n}^{2}\left|\hat{K}_{+}(-\nu)-\hat{K}_{-}(-\nu)\right|\left|f_{n}(\tau)\right|}{\left(\nu^{2}+\delta^{2} a_{n}^{2}\right)^{2}} d \nu=I_{1 n}+I_{2 n} .
$$

Разобьем интеграл в правой части (58) на два интеграла: от $\varepsilon>0$ до $+\infty$ и от 0 до $\varepsilon$. Первый интеграл обозначим $I_{1 n}$, второй интеграл обозначим $I_{2 n}$. Перейдем к оценке интеграла $I_{1 n}$.

Заметим, что для любого $\varepsilon>0$ для всех $\nu \in(\varepsilon,+\infty)$ имеет место оценка

$$
\frac{a_{n}^{2}}{\nu^{2}+\delta^{2} a_{n}^{2}} \leqslant d_{2},
$$

где $d_{2}$ - положительная постоянная, не зависящая от $n$. 
На основании (54)-(59), получаем

$$
I_{1 n} \leqslant \int_{\varepsilon}^{+\infty} e^{-\nu(t-\tau)} \frac{a_{n}^{2}\left|\hat{K}_{+}(-\nu)-\hat{K}_{-}(-\nu)\right|\left|f_{n}(\tau)\right|}{\left(\nu^{2}+\delta^{2} a_{n}^{2}\right)^{2}} d \nu \leqslant e^{-\varepsilon(t-\tau)} q_{1}\left(\int_{\varepsilon}^{+\infty} \frac{d \nu}{\tau^{\alpha}\left(\nu^{2}+\delta^{2} a_{n}^{2}\right)}\right)\left|f_{n}(\tau)\right|
$$

с положительной постоянной $q_{1}$, не зависящей от $n$.

Осуществив замену переменных $\eta=\nu / a_{n}$, получаем, что

$$
\int_{\varepsilon}^{+\infty} \frac{d \nu}{\nu^{\alpha}\left(\nu^{2}+\delta^{2} a_{n}^{2}\right)}=\frac{1}{a_{n}^{1+\alpha}} \int_{\varepsilon / a_{n}}^{+\infty} \frac{d \eta}{\eta^{\alpha}\left(\delta^{2}+\eta^{2}\right)} \leqslant \frac{1}{a_{n}^{1+\alpha}} \int_{0}^{+\infty} \frac{d \eta}{\eta^{\alpha}\left(\delta^{2}+\eta^{2}\right)} .
$$

В силу того, что $\alpha \in(0,1 / 2)$ интегралы в правой части (61) являются сходящимися. Из оценок (60) и соотношения (61) вытекает, что

$$
I_{1 n} \leqslant q_{2} e^{-\varepsilon(t-\tau)}\left|a_{n}^{-(1+\alpha)} f_{n}(\tau)\right|
$$

с положительной постоянной $q_{2}$, не зависящей от $n$.

Перейдем к оценке интеграла $I_{2 n}$. Интеграл $I_{2 n}$ может быть переписан в виде

$$
I_{2 n}=\int_{0}^{\varepsilon} e^{-\nu(t-\tau)} \frac{\left|a_{n}^{-2} f_{n}(\tau)\right|\left|\hat{K}_{+}(-\nu)-\hat{K}_{-}(-\nu)\right|}{\left(\nu^{2} / a_{n}^{2}+1\right)^{2}} d \nu
$$

Заметим, что при малых $\tau \in(0, \varepsilon)$, согласно (54)

$$
\left|\hat{K}_{+}(-\nu)-\hat{K}_{-}(-\nu)\right| \leqslant p_{1} \nu^{\alpha}
$$

с положительной постоянной $p_{1}$. Отсюда, из $(63),(64)$ следует, что $I_{2 n}$ допускает оценку

$$
I_{2 n} \leqslant p_{2} \int_{0}^{\varepsilon} e^{-\nu(t-\tau)} \nu^{\alpha}\left|a_{n}^{-2} f_{n}(\tau)\right| d \nu=p_{2} J_{2 n}
$$

с положительной постоянной $p_{2}$, не зависящей от $n$.

Сделаем замену переменных $\theta=\nu(t-\tau), d \theta=(t-\tau) d \nu$. Тогда интеграл в правой части (65) примет вид

$$
J_{2 n}=\frac{1}{(t-\tau)^{1+\alpha}} \int_{0}^{\varepsilon(t-\tau)} \theta^{\alpha} e^{-\theta}\left|a_{n}^{-2} f_{n}(\tau)\right| d \theta .
$$

Применяя теперь к интегралу в правой части (66) вторую теорему о среднем, получим

$$
J_{2 n}=\frac{(\varepsilon(t-\tau))^{\alpha}}{(t-\tau)^{1+\alpha}} \int_{\xi_{1}}^{\varepsilon(t-\tau)} e^{-\theta}\left|a_{n}^{-2} f_{n}(\tau)\right| d \theta, \quad \xi_{1} \in(0, \varepsilon(t-\tau))
$$

В свою очередь интеграл в правой части соотношения (67) допускает следующую оценку:

$$
J_{2 n} \leqslant \frac{(\varepsilon(t-\tau))^{1+\alpha}\left|a_{n}^{-2} f_{n}(\tau)\right|}{(t-\tau)^{1+\alpha}}=\varepsilon^{1+\alpha}\left|a_{n}^{-2} f_{n}(\tau)\right| .
$$

Отсюда из соотношений (65) и (68) получаем оценку

$$
I_{2 n} \leqslant q_{3} \varepsilon^{1+\alpha}\left|a_{n}^{-2} f_{n}(\tau)\right|
$$

с положительной постоянной $q_{3}$, не зависящей от $n$.

Объединяя оценки (58), (62) и (69), в итоге получаем

$$
\left|u_{R n}(t, \tau)\right| \leqslant q_{2} e^{-\varepsilon(t-\tau)}\left|a_{n}^{-1-\alpha} f_{n}(\tau)\right|+q_{3} \varepsilon^{1+\alpha}\left|a_{n}^{-2} f_{n}(\tau)\right|
$$

с положительными постоянными $q_{2}$ и $q_{3}$, не зависящими от $n$. 
Из оценки (70) вытекает оценка вектор-функции $v_{R}(t, \tau)$ : В свою очередь, оценка (71) может быть переписана в виде

$$
\left\|v_{R}(t, \tau)\right\|^{2} \leqslant k_{2} e^{-2 \varepsilon(t-\tau)}\left\|A^{-1-\alpha} f(\tau)\right\|^{2}+k_{3} \varepsilon^{2(1+\alpha)}\left\|A^{-2} f(\tau)\right\|^{2} .
$$

Из последней оценки вытекает следующая цепочка неравенств

$$
\begin{aligned}
\left\|u_{R}(t)\right\|^{2}=\| \int_{0}^{t} v_{R}(t, \tau) d \tau & \left\|^{2} \leqslant\left(\int_{0}^{t}\left\|v_{R}(t, \tau)\right\| d \tau\right)^{2} \leqslant t \int_{0}^{t}\right\| v_{R}(t, \tau) \|^{2} d \tau \leqslant \\
& \leqslant t\left\{k_{2} \int_{0}^{t} e^{-2 \varepsilon(t-\tau)}\left\|A^{-1-\alpha} f(\tau)\right\|^{2} d \tau+k_{3} \varepsilon^{2(1+\alpha)} \int_{0}^{t}\left\|A^{-2} f(\tau)\right\|^{2} d \tau\right\} .
\end{aligned}
$$

Наконец, объединяя оценки (51) и (72), получаем следующую оценку решения:

$$
\begin{aligned}
& \|u(t)\| \leqslant d_{1} t \int_{0}^{t}\left\|Q_{n_{0}} e^{-k A^{1-\alpha}(t-\tau)} A^{-1} f(\tau)\right\|^{2} d \tau+d_{2} t \int_{0}^{t} e^{-2 \delta(t-\tau)}\left\|P_{n_{0}} A^{-1} f(\tau)\right\|^{2} d \tau+ \\
& \quad+t\left\{k_{1} \int_{0}^{t} e^{-2 \varepsilon(t-\tau)}\left\|A^{-(1+\alpha)} f(\tau)\right\|^{2} d \tau+k_{2} \varepsilon^{2(\alpha+1)} \int_{0}^{t}\left\|A^{-2} f(\tau)\right\|^{2} d \tau\right\}, \quad t>0
\end{aligned}
$$

Оценки (23) для $m=1,2$ немедленно вытекают из полученной оценки (73), если в оценке (73) $f(\tau)$ и $u(t)$ заменить на $A^{m} f(\tau)$ и $A^{m} u(t)$, соответственно $(m=1,2)$. Поскольку в процессе доказательства теоремы 8 мы работаем со скалярными функциями $f_{n}(\tau)=\left(f(\tau), e_{n}\right), u_{n}(t)=$ $\left(u(t), e_{n}\right)$, замена $f_{n}(\tau), u_{n}(t)$ на $a_{n}^{m} f_{n}(\tau), a_{n}^{m} u_{n}(t)$ происходит по существу автоматически, как показывает анализ доказательства теоремы 8.

Теорема 8 доказана.

\section{СПИСОК ЛИТЕРАТУРЫ}

1. Власов В. В., Гавриков А. А., Иванов С. А., Князъков Д. Ю., Самарин В. А., Шамаев А. С. Спектральные свойства комбинированных сред// Совр. пробл. мат. мех. - 2009. - 5, № 1. - С. 134-155.

2. Власов В. В., Раутиан Н. А. О свойствах решений интегродифференциальных уравнений, возникающих в теории тепломассообмена// Тр. Моск. мат. о-ва. - 2014. - 75, № 2. - С. 131-155.

3. Власов В. В., Раутиан Н. А. Корректная разрешимость и спектральный анализ интегродифференциальных уравнений, возникающих в теории вязкоупругости// Совр. мат. Фундам. напр. - 2015. 58. - C. 22-42.

4. Власов В. В., Раутиан Н. А. Корректная разрешимость вольтерровых интегро-дифференциальных уравнений в гильбертовом пространстве// Диффер. уравн. - 2016. - 52, № 9. - С. 1168-1177.

5. Власов В. В., Раутиан Н. А. Спектральный анализ функционально-дифференциальных уравнений. - M.: MAKC Пресc, 2016.

6. Власов В. В., Раутиан Н. А. Исследование операторных моделей, возникающих в теории вязкоупругости// Совр. мат. Фундам. напр. - 2018. - 64, № 1. - С. 60-73.

7. Власов В. В., Раутиан Н. А. Корректная разрешимость и спектральный анализ вольтерровых интегродифференциальных уравнений с сингулярными ядрами// Докл. РАН. - 2018. - 482, № 6 . C. $635-638$.

8. Власов В. В., Раутиан Н. А. Корректная разрешимость и представление решений вольтерровых интегро-дифференциальных уравнений, вонизникающих в теории вязкоупругости// Диффер. уравн. -2019 . - 55, № 4. - C. 574-587.

9. Власов В. В., Раутиан Н. А. Исследование операторных моделей, возникающих в задачах наследственной механики // Тр. семин. им. И. Г. Петровского. - 2019. - 32. - С. 91-110.

10. Власов B. B., Раутиан H. A. Спектральный анализ и представление решений интегродифференциальных уравнений с дробно-экспоненциальными ядрами// Тр. Моск. мат. о-ва. - 2019. - 11. - C. $41-56$. 
11. Жиков В. В. Об одном расширении и применении метода двухмасштабной сходимости// Мат. сб. 2000. - 191, № 7. - С. 31-72.

12. Илъюшин A. А., Победря Б. Е. Основы математической теории термовязкоупругости. - М.: Наука, 1970.

13. Льков А. В. Проблема тепло- и массообмена. - Минск: Наука и техника, 1976.

14. Работнов Ю. Н. Элементы наследственной механики твердых тел. - М.: Наука, 1977.

15. Amendola G., Fabrizio M., Golden J. M. Thermodynamics of Materials with Memory. Theory and Applications. - New York: Springer, 2012.

16. Desch W., Miller R. Exponential stabilization of Volterra Integrodifferential equations in Hilbert space// J. Differ. Equations. — 1987. — 70. - P. 366-389.

17. Eremenko A., Ivanov S. Spectra of the Gurtin-Pipkin-Type Equations// SIAM J. Math. Anal. — 2011. 43, № 5. - P. 2296-2306.

18. Gurtin M. E., Pipkin A. C. Theory of heat conduction with finite wave speed// Arch. Rat. Mech. Anal. 1968. - 31. - P. 113-126.

19. Lions J. L. and Magenes E. Nonhomogeneous Boundary-Value Problems and Applications. - BerlinHeidelberg-New York: Springer-Verlag, 1972.

20. Pandolfi L. The controllability of the Gurtin-Pipkin equations: a cosine operator approach// Appl. Math. Optim. - 2005. - 52. - P. 143-165.

21. Prüss J. Evolutionary Integral Equations amd Applications. - Basel-Baston-Berlin: Birkhäuser, 1993.

22. Vlasov V. V., Rautian N. A. Well-deffined solvability and spectral analysis of abstract hyperbolic equations// J. Math. Sci. - 2011. - 179, № 3. - P. 390-415.

23. Vlasov $V . V ., W u$ J. Solvability and spectral analysis of abstract hyperbolic equations with delay// Funct. Differ. Equations. - 2009. — 16, № 4. - P. 751-768.

24. $W u$ J. Semigroup and integral form of class of partial differential equations with infinite delay// Differ. Integral Equations. — 1991. — 4, № 6. - P. 1325-1351.

Власов Виктор Валентинович

Московский государственный университет им. М. В.Ломоносова

E-mail: vikmont@yandex.tu.

Раутиан Надежда Александровна

Московский государственный университет им. М. В. Ломоносова

E-mail: nrautian@mail.ru 